\title{
HYPERTHYROIDISM AS A DIFFERENTIAL DIAGNOSIS OF MANIFESTATIONS OF SYSTEMIC LUPUS ERYTHEMATOSUS
}

Raissa Velasques de Figueiredo ${ }^{1, *}$, Maria Lucia Lemos Lopes ${ }^{1}$, Tatiana Freitas Tourinho ${ }^{1}$, Gilberto Scanagatta ${ }^{1}$, Rafael Coradin ${ }^{1}$, Thiago Willers ${ }^{1}$, Eduardo Rosa de Oliveira ${ }^{1}$, Gabriela Sasso Padilha ${ }^{1}$, Bruno Trevisan ${ }^{1}$, Maria Odete Esteves Hilario ${ }^{1}$, Luana Ribeiro Carlos $^{1}$, Fabiana Jaeger ${ }^{1}$

1. Universidade Federal de Ciências da Saúde de Porto Alegre, Porto Alegre (RS), Brasil.

*Corresponding author: consultoriowillers@gmail.com

\section{BACKGROUND}

Hyperthyroidism is an uncommon thyroid disorder. Its prevalence among lupus patients is not greater than the general population and its symptoms can mimic well-known symptoms of the rheumatologist's daily practice.

\section{CASE REPORT}

A 29-year-old female patient, dark-skinned, diagnosed with systemic lupus erythematosus (SLE) with a fine speckled nuclear reagent 1:640 ANA, arthritis, photosensitivity, alopecia and thrombocytopenia. Active smoker and history of previous cholecystectomy. The patient seeks an assistant team with severe nausea and vomiting (about10 episodes a day), unrelated to food, without abdominal pain or changes in bowel habits for about 60 days. She reported weight loss of $10 \mathrm{~kg}$ in those 2 months. She denied jaundice and pathological elements during emesis. In the previous week, dyspnea on medium exertion had started with progressive worsening, associated with edema of the lower limbs and precordial discomfort "in a sting", constant, without irradiation, without changing the pain pattern with a change in position. Physical examination showed tachycardia (FC 120) with a regular rhythm. The hypothesis of myocarditis was suggested, which could even be postinfectious (GIT infection causing vomiting). The tests requested showed thrombocytopenia (40,000), hemoglobin 10 anemia, with no changes in CK-MB or troponin. Transthoracic echocardiography did not show pericardial effusion or myocardial dysfunction, it showed an increase in pulmonary artery caliber. Computed tomography angiography was performed and ruled out pulmonary thromboembolism. Hemolysis was not found. Believing that the symptoms were triggered by the underlying disease, we chose to start prednisone $1 \mathrm{mg} / \mathrm{kg} /$ day. During the laboratory investigation, TSH and free T4 were requested, which were altered, with TSH 0.01 (0.55-4.78) Free T42.24 (0.89-1.76) Free T3 5.46 (2.3-4.2). It was evident that the condition was decompensated hyperthyroidism, and treatment with propranolol and tapazole was started. With gradual improvement of symptoms.

\section{CONCLUSION}

Symptoms improved after the treatment of hyperthyroidism. A literature review showed that the symptoms would be secondary to this thyroid disorder, what was confirmed with the improvement after the treatment. This report aims to remember that hyperthyroidism, although less common, can cause symptoms that confuse us in the differential diagnosis of lupus patient. 